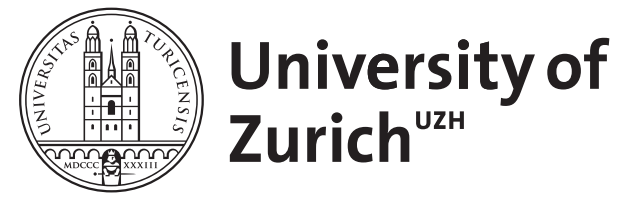

\title{
Quantitative Aspekte der bildgebenden Kernresonanz
}

Bösiger, $\mathrm{P}$

DOI: https://doi.org/10.1515/bmte.1988.33.s2.143

Posted at the Zurich Open Repository and Archive, University of Zurich ZORA URL: https://doi.org/10.5167/uzh-153923

Journal Article

Published Version

Originally published at:

Bösiger, P (2009). Quantitative Aspekte der bildgebenden Kernresonanz. Biomedizinische Technik. Biomedical engineering, 33(Suppl 2):143-146.

DOI: https://doi.org/10.1515/bmte.1988.33.s2.143 


\title{
Quantitative Aspekte der bildgebenden Kernresonanz
}

\author{
P. Bösiger
}

Institut für Biomedizinische Technik und Medizinische Informatik, Universität und ETH Zürich

\section{Einleitung}

Seit ihrer Entdeckung durch F. Bloch und E. Purcell im Jahre $19+6$ werden die magnetischen Kernresonanzverfahren unter ständiger Weiterentwicklung in der Chemie und der Physik eingesetzt hauptsächlich für die Aufklärung der Strukturen von Molekülen und Kristallgittern, zur Analyse von chemischen Lösungen und für Untersuchungen des Ablaufs von chemischen Reaktionen. Mit zunehmendem Interesse an der Chemie und der Physik von biologischen Prozessen werden sie seit rund 20 Jahren auch zum Studium von organisierten biologischen Systemen beigezogen. Sie liefern wertvolle Erkenntnisse über Stoffwechselprozesse, sowohl von einfachen Organismen als auch von isolierten Organen höherer Lebewesen. Seit 1972 sind Techniken in Entwicklung (1-3), die eine räumliche Zuordnung der Kernresonanzsignale innerhalb der untersuchten Strukturen zulassen. Doch erst mit dem Bau von Ganzkörpermagneten wurden die Verfahren von grundlegendem Interesse für die Medizin, ermöglichen sie doch praktisch risikofrei nichtinvasive Untersuchungen von strukturellen Veränderungen und von biochemischen Vorgängen im Innern des menschlichen Körpers.

Die Kernspintomographie, auch als Magnetic Resonance Imaging (MRI) bezeichnet, ist heute technisch soweit entwickelt, dass sie in zunehmendem Masse in der Diagnostik nutzbringend eingesetzt wird. Sie erzeugt Schnittbilder durch den menschlichen Körper in beliebigen Richtungen, insbesondere auch parallel zur Körperachse. Im Gegensatz zur Röntgentomographie oder zur Ultraschallechographie, bei denen Absorptionskoeffizienten der Röntgenstrahlung oder Streueigenschaften des Gewebes für Ultraschallwellen im Bild wiedergegeben werden, gewinnt man bei der Kernspintomographie Informationen über die Verteilungsdichten und die Relaxationszeiten der den medizinischen Anwendungen besonders leicht zugänglichen Wasserstoffkerne. Die Vorteile der Kernspintomographie im Vergleich zu den konventionellen bildgebenden Verfahren sind heute neben der Risikofreiheit und der beliebigen Lage der Schnittebenen ein wesentlich besserer Weichteilkontrast und das Fehlen jeglicher Knochenartefakte. Die Bilder erlauben deshalb eine sehr differenzierte Beurteilung der anatomischen Strukturen im Körperinnern.

Die magnetische Kernresonanz (4) basiert auf der Tatsache, dass eine Reihe von Atomkernen wie ${ }^{1} \mathrm{H},{ }^{13} \mathrm{C},{ }^{23} \mathrm{Na},{ }^{31} \mathrm{P}$ u.a.m. einen Eigendrehimpuls oder Spin aufweisen [6]. Gekoppelt mit diesem Drehimpuls zeigen die Kerne ein kleines magnetisches Dipolmoment. Unter der Wirkung eines äusseren Magnetfeldes richten sich diese nuklearen Dipolmomente unter ständiger Präzession um die Feldrichtung $\omega_{0}=\gamma B_{0}$ mit der sog. Larmorfrequenz vorzugsweise parallel zu dieser Feldrichtung aus. $B_{o}$ stellt das Magnetfeld am Ort des Kernes dar; $\boldsymbol{\gamma}$ ist eine kernspezifische Proportionalitătskonstante, das gyromagnetische Verhältnis. Dadurch entsteht in einer Probe ein makroskopisches Dipolmoment, die sogenannte Magnetisierung. Beim Anlegen eines Rädiowellenpulses, dessen Frequenz exakt mit der Larmorfrequenz der Kernmomente übereinstimmt, tritt ein Resonanzphänomen auf, das die Magnetisierung aus der Gleichgewichtslage wegdreht. Durch sorgfältige Wahl der Amplitude und der zeitlichen Dauer des Pulses tritt beispielsweise eine Drehung um $90^{\circ}$ oder um $180^{\circ}$ auf. Nach einem $90^{\circ}$-Puls präzessiert die Magnetisierung in einer Ebene senkrecht zum äusseren Feld und induziert in der Empfängerspule ein Signal. Dieses Signal verschwindet wieder, infolge von Relaxationseffekten mit Zeitkonstanten $T_{1}$ und $T_{2}$, die charakteristisch sind für die verschiedenen Arten von Gewebe. Die räumliche Zuordnung der Signale wird durch sogenannte Gradientenfelder vorgenommen, welche das statische Magnetfeld örtlich verstärken oder abschwächen. Dadurch wird die Larmorfrequenz abhängig von der räumlichen Koordinate des Objektes. Durch Einschalten von Gradientenfeldern ' in allen drei Raumrichtungen nacheinander lassen sich die Signale den entsprechenden Raumkoordinaten zuordnen.

Quantitative Gewebecharakterisierung bei pionenbestrahlten Hirntumoren

Die Amplitude der Signale und damit die Grauwerte der Bildpunkte hängen im allgemeinen in komplizierter Weise von der. Dichte der zur Abbildung im allgemeinen beigezogenen Wasserstoffkerne des '”, entsprechenden 
Gewebes, von den Relaxationszeiten dieser Kerne und von einer Reihe experimentell wählbarer Parameter ab, welche die Anregungspulssequenz definieren. Eine quantitative Charakterisierung des Gewebes ist nur möglich aufgrund von Bildern, auf denen die gewebespezifischen Spindichten $\rho$ oder die Relaxationszeiten $T_{1}$ oder $T_{2}$ allein als Grauwerte kodiert dargestellt sind. Die Acquisition von Daten, aus denen die Berechnung von $\rho, T_{1}$ und $T_{2}$ mit hoher Genauigkeit möglich sind, stösst insofern auf Probleme, als sie die Untersuchungszeit für den Patienten wesentlich erhöht. Eine Lösung dieses Problems stellt die Multiple Saturation-Recovery-Sequenz dar (5), die den Wiederaufbau der Magnetisierung nach der Sättigung in einer wählbaren Anzahl von Messpunkten dokumentiert. Daraus können $T_{1}$ Bilder berechnet werden. Der zusätzliche Einbau einer Reihe von Spin-Echo-Pulsen in das letzte SaturationRecovery-Segment ermöglicht ohne Verlängerung der Messzeit die Berechnung von $\rho$ - und $T_{2}$-Bildern.

Die Sequenz wird in einer Pilotstudie eingesetzt zur Dokumentation der Gewebeveränderung von Hirntumoren (Astrozytomen), die am Paul Scherrer-Institut in der Schweiz mit Pionen bestrahlt werden (6). Die Patienten werden vor, während und periodisch nach der Therapie mittels MRI untersucht, wobei die Relaxationszeiten $T_{1}$ und $T_{2}$ bildlich aufgezeichnet und deren Verlauf im Tumor, im den Tumor umgebenden Oedem, im gesunden Gewebe nahe beim Oedem, das mit einer Dosis von $90 \%$ bestrahlt wird, und an der zum Tumor kontralateralen Stelle festgehalten werden. Diese Relaxationszeiten stehen in enger Korrelation mit dem Wassergehalt des Gewebes und damit beispielsweise zum Schweregrad des Oedems. In Ergänzung werden mit konventioneller Multislice-Spin-Echo-Technik Bilder erzeugt, welche eine visuelle Beurteilung des Therapieverlaufs und eine Quantifizierung der Tumor- und Oedemvolumina zulassen.
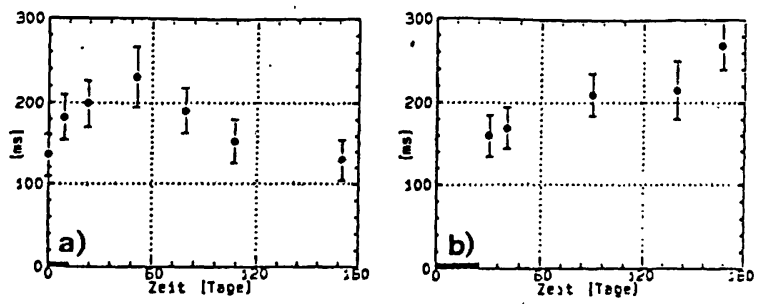

Abb.1: Relaxationszeiten $T_{2}$ von Tumoren vor, während und nach der zwei- bis vierwöchigen Bestrahblung

Wenn auch zur Zeit erst drei Patienten länger als ein Jahr untersucht wurden, zeichnen sich interessante Ergebnisse ab: Bei allen Tumoren und bei den Oedemen sind die Relaxationszeiten $T_{1}$ und $T_{2}$ bereits vor Beginn der Bestrahlung höher als im gesunden Gewebe. Während der Bestrahlungsperiode und zwei bis drei Monate danach steigen sie weiter an. Dies lässt sich damit erklären, dass die Lysosomenmembranen zerstört und damit hydrolytische Enzyme freigesetzt werden, wodurch der Wassergehalt des Gewebes ansteigt.
Nach dieser Periode werden zwei verschiedene Arten von Weiterentwicklung beobachtet. Bei 5 Patienten fallen die die kelaxationszeiten kontinuierlich ab gegen oder sogar unter Verhalten widerspiegelt der Bestralilung (Abb. 1a). Das tionsvorgängen, bei denen Einsetzen von Reparazerstörte Parenchym durch Bindegewebe die Bestrahlung Gleichzeitig nimmt darch Bindegewebe ersetzt wird. auf einige diese Patienten erscheint Anfangswertes. Die Prognose für ihre berufliche Tïligkeit vieder aufgenommen. Der Verlauf reflektiert offensichtlich eine mindestens temporäre Kontrolle des Tumorwachstums.

Bei den anderen Fällen wird ein kontinuierliches Anwachsen von $T_{1}$ und $T_{2}$ des Tumors und des Oedems festgestellt (Abb. 1b). Gleichzeitig steigt das Oedemvolumen an. Die Prognose dieser Patienten erwies sich als ungünstig; die Patienten starben innerhalb der folgenden Wochen.

Die sorgfältige Analyse der Daten zeigt ausserdem, dass das Verhalten der Oedeme korreliert mit dem Verhalten der Tumore, wenn auch die Veränderungen von $T_{1}$ und $T_{2}$ der Oedeme in den meisten Fällen weniger ausgeprägt sind. Die Relaxationszeiten des gesurden Gewebes hingegen werden durch die Bestrahlung nicht beeinflusst, auch wenn dieses Strahlendosen von bis zu $90 \%$ der Dosis des Tumors erfährt. Daraus kënnte geschlossen werden, dass der Wassergehalt des oedematösen Gewebes nicht durch die Strahlung selbst beeinflusst wird, sondern eine Folge der Reaktion des Tumors auf die Bestrahlung darstellt. Die Tatsache, dass sich die Relaxationszeiten des gesunden Gewebes auch bei $90 \%$ Bestrahlung kaum verändern, zeigt ausserdem, dass die Strahlentoleranz von $T_{1}$ und $T_{2}$ des gesunden Gewebes für Pionen höher ist als die des Tumors.

\section{Quantitative Blutflussmessungen}

Quantitative Information über die Bewegung von Strukturen, insbesondere über die Blutflussgeschuindigkeit in grösseren Gefässen, lässt ich aus den bildlich dargestellten Phasen der Kernresonanzsignale ableiten (7). Die bei Spin-EchoSequenzen infolge eines magnetischen Feldgradienten $G_{2}$ durch den Blutfluss in $z$-Richtung induzierte Phasenverschiebung $\Delta \phi$ beträgt

$$
\begin{array}{ll}
\Delta \phi= & 1 / 4 \gamma \mathrm{G}_{2} v_{2} T_{\mathrm{E}}{ }^{2} \\
\gamma & \text { gyromagnetisches Verhältnis } \\
v_{2} & \text { Geschwindigkeit des Blutflusses } \\
\mathrm{T}_{\mathrm{E}} & \text { Echozeit. }
\end{array}
$$

Zur Analyse von pulsatilem Fluss werden auf einem Philips Gyroscan S15 (1.5T) schnelle Pulssequenzen eingesetzt, bei denen die Spinechos durch Gradienteninversion entstehen (FFE-Sequenzen)(8). Sic erlauben bei Trigserung der Messzyklen auf das EKG stroboskopisch die Erzeugung on bis zu 64 Momentanbildern innerhalb des Herzanklus. Aus den entsprechenden Phasenbildern kann unter detaillierter Kenntnis der Gradientensequenz die instantane Blutflussgeschwindigheit räumlich aufgelint utor den Gefässquerschnitt ermittelt werden (Abb.2). 


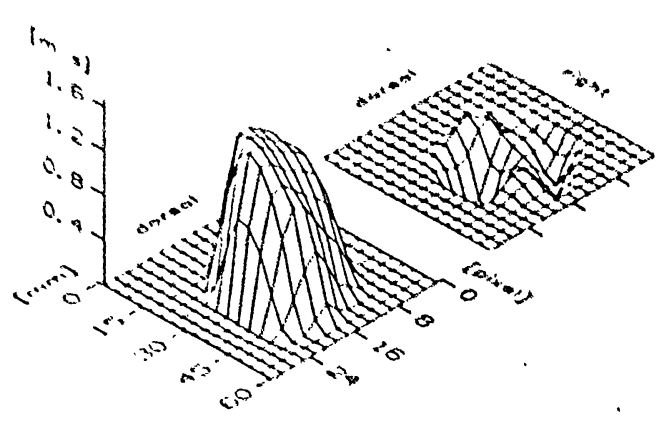

Ath. 2: Systolische und pextsystolische Blutgeschwindigkeitsprofile in der aufscizgenden Aorta.

Die Integration über den Querschnitt führt zum instantanen Blutfluss; die zeitliche Mittelung dieser Instantanwerte über einen Herzzyklus erlaubt die Bestimmung des mittleren Blutflusses pro Herzzyklus. Die Technik wird erfolgreich eingesetzt zur Blutflussbestimmung in der Aorta ascendens und in der Aorta abdominalis von gesunden Probanden (Abb. 2) und von Patienten mit Herzklappeninsuffizienzen. Die entsprechenden bildpunktweise über den Gefässquerschnitt aufgezeichneten Flussgeschwindigkeitsprofile erlauben eine sehr detaillierte Analyse des Flusses. Die Profile zu einem Film aneinandergereiht ergeben eine eindrückliche Darstellung der Dynamik des Blutflusses. Deutlich erkennbar ist der Rückfluss in der Postsystole und die anschliessende dichrotische Welle, welche durch die Reflexion des Pulses an der Peripherie entsteht. Der Vergleich mit Ultraschall-Dopplermessungen zeigt eine weitgehende Übereinstimmung der Dynamik und der über den Herzzyklus gemittelten Flusswerte. Er zeigt aber auch die Grenzen der Ultraschall-Doppler-Technik, wenn bei der Flussbestimmung zylindersymmetrische Flussverhältnisse vorausgesetzt werden.

$\mathrm{Zu}$ ergänzen bleibt, dass derartige quantitative Blutflussmessungen mit konventionellen FFE-Sequenzen wegen der unvermeidlich auftretenden Phasenfeldern im Bild beschränkt sind auf Gefässe, bei denen während einer gewissen Zeitspanne in der Diastole kein Fluss vorhanden ist. Die Bilder dieser Phase werden als Referenzbilder verwendet. Für Flussmessungen an andern Gefässen sind gegenwärtig weitere Puls- und Gradientensequenzen in Entwicklung.

\section{Chemische Verschiebung und lokale Spektroskopie}

Bisher wurde angenommen, dass abgesehen von stochastischen Dephasierungseffekten gleiche Kerne identische Larmorfrequenzen aufweisen. Sie alle haben das gleiche gyromagnetische Verhältnis, was zu identischen Präzessionsfrequenzen führt, sofern sie das gleiche lokale Magnetfeld verspüren. Die Elektronen des Atoms aber, die den Kern umkreisen, schirmen das äussere Magnetfeld leicht $a b$, so dass das Kernmoment der Wirkung eines leicht schwächeren Feldes ausgesetzt wird. Die Larmorfrequenz wird abhängig von der Elektronenkonfiguration und damit von Jer molekularen Struktur, in, welche das Atom eingebaut ist. Die Kerne von identischen Atomen also, welche In verschiedenen Molekulen eingebaut sind oder welche verschiedene Plätze im selben Molekul besetzen, senden nach der Anregung Signale leicht verschiedener Frequenzen aus. NMR-Spektren von Proben, in denen die untersuchten Atome in verschiedenen Verbindungen vorliegen, zeigen mehrere Resonanzlinien. Die Frequenzverschiebungen im Vergleich zu den Resonanzlinien von Referenzsubstanzen werden in parts per million (ppm) angegeben und als chemische Verschiebung bezeichnet.

In der NMR-Spektroskopie am Lebewesen stellt sich die Frage, wie solche Spektren auf ausgewählten Körperstellen lokalisiert werden können. Am einfachsten erfolgt die Volumenselektion durch sogenannte Oberflächenspulen. Sie werden auf der Körperoberfläche direkt über dem zu untersuchenden Bereich angebracht und zeichnen sich durch einen unscharf begrenzten halbkreisförmigen Empfindlichkeitsbereich aus. Er kann durch geeignete Wahl der Pulssequenzen, die für die Anregung der Kerne verwendet werden, weiter eingeschränkt werden. Die Vorteile der Oberflächenspulen liegen in der hohen Empfindlichkeit, die entscheidenden Nachteile bilden aber die Beschränkung der Lokalisation auf oberflächenah gelegene Körperstrukturen und die Ungenauigkeit der Lokalisation, indem der auf dem Magnetresonanzbild identifizierte Bereich nicht direkt auf den zu untersuchenden Patienten übertragen wird.

Die Nachteile können durch eigentliche volumenselektive Messverfahren behoben werden, bei denen die Selektion eines ausgewählten Volumens aufgrund von Kombinationen komplexer Anregungspulssequenzen mit entsprechenden Gradientensequenzen erfolgt. Verschiedene Techniken wurden dazu vorgeschlagen, die unter Namen wie VSE (volume selective excitation) (9), SPARS (spatially resolved spectroscopy) (10), ISIS (image selected in vivo spectroscopy) (11), STEVE (stimulated echo volume excitation), sowie VSR (volume selective refocussing) (12) u.a.m. bekannt sind.

VSR hat sich für die ${ }^{1} \mathrm{H}$-Spektroskopie sehr bewährt. Es zeichnet sich aus durch hohe Unterdrückung von Signalen ausserhalb des selektiven : Volumens und durch einfache Anwendung am lebenden Objekt. Ausserdem lässt es sich mit Verfahren kombinieren, die aus der Laborspektroskopie bekannt sind zur Unterdrückung der Resonanzsignale von Wasser oder von Fett, die im Gewebe in so hohen Konzentrationen vorhanden sind, dass die Signale weiterer ${ }^{1} \mathrm{H}$-Verbindungen überdeckt werden. Auf diese .Verfahren, die unter den Namen Binomialpulse und Multi Quantum Filtering bekannt sind, soll hier nicht näher eingegangen werden, da sie in einem separaten Beitrag (13) behandelt werden.

ISIS wird heute hauptsächlich für die Lokalisation bei der ${ }^{31}$ P-Spektroskopie eingesetzt. Die Anregungspuls- und Gradientensequenz ist in Abb.3 dargestellt. Betrachten wir vorerst nur den ersten Teil der Präparationsperiode und die Ausleseperiode: Ein schmalbandiger $180^{\circ}$-Puls zusammen 
mit dem Gradienten $\mathbf{G}_{\mathbf{x}} \cdot$ invertiert die Magnetisierung einer zu einer $x$-Achse orthogonalen Schicht der Probe, weil nur in dieser Schicht die Resonanzbedingung erfültt ist. Ausserhalb dieser Schicht liegt wegen dem Gradientenfeld ein stärkeres oder ein schwächeres Magnetfeld vor. Die Resonanzbedingung ist nicht erfüllt, die magnetischen Momente bleiben unbeeinflusst. Nach Anregung der gesamten Probe durch einen $90^{\circ}$-Puls werden die Signale ausgelesen. Dabei zeigen die Signale der Magnetisierung der Schicht, welche vorher durch den $180^{\circ}$-Puls invertiert wurde, ein negatives Vorzeichen. Dasselbe Experiment wird nun zweimal nacheinander durchgeführt, das eine $\mathrm{Mal}$ mit, das zweite Mal ohne den schichtselektiven Inversionspuls. . Nach der Subtraktion der beiden Signale bleibt nur das Signal der selektierten Schicht.

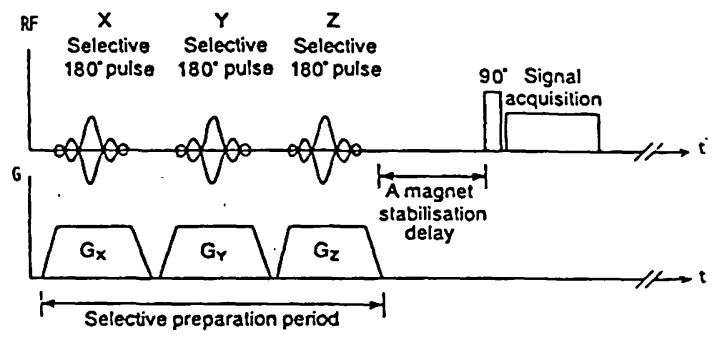

Abb. 3: Puls- und Gradientensequenz für ISIS.

Die Sequenz kann für die Begrenzung des Volumens in allen drei Raumrichtungen erweitert werden durch Hinzufügen von zwei mit entsprechenden Feldgradienten in den andern beiden Richtungen selektive $180^{\circ}$-Pulse. Das Experiment wird dann mit einer Phase Cycling Periode von acht durchgeführt, d.h. es wird acht mal durchgeführt mit allen möglichen Kombinationen der drei Pulse ein- und ausgeschaltet. Die resultierenden Signale sind anschliessend so zu addieren und zu subtrahieren, dass für die Signale ausserhalb des ausgewählten Volumens eine destruktive Interferenz auftritt. Der Vorteil von ISIS in der ${ }^{31}$ P-Spektroskopie liegt darin, dass die Signalabtastung unmittelbar nach dem $90^{\circ}$-Anregungspuls erfolgen kann, wodurch die Signalverluste infolge der $T_{2}$-Relaxation trotz der relativ kurzen Relaxationszeiten der ${ }^{31}$ P-Kerne bescheiden bleiben.

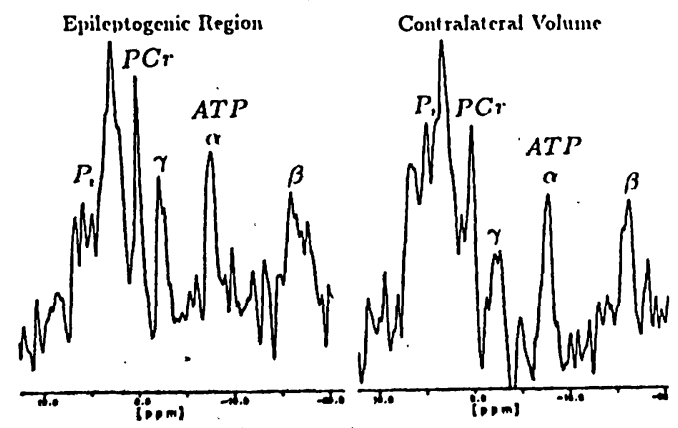

Abb. 4: ${ }^{31}$-P.Spektren eines Patienten mit Epilepsie aus dem Gebiet des Fokus (links) und aus dem kontralateralen Volumen (rechts).
Abb.4 zeigt zwei ${ }^{31}$ P-Spektren eines Patienten mit einer epileptischen Erkrankung (14). Das eine Spektrum aus einem Volumen von etwa $120 \mathrm{~cm}^{3}$ Grösse stammt aus dem Gebiet, wo im Bild der Fokus erkennbar ist. Das andere Spektrum wurde kontralateral in einem Kontrollvolumen aufgenommen. Auf beiden Spektren erkennt man von rechts nach links drei Resonanzlinien des Adenosintriphosphates (ATP), dann eine Linie des Kreatinphosphates ( $\mathrm{PCr}$ ) und die drei sich überlagernden Linien von Phosphodiestern, des anorganisch gebundenen Phosphors $\left(\mathrm{P}_{\mathrm{i}}\right)$ und von Phosphomonoestern. Während derartige Spektren bei gesunden Probanden weitgehend identisch aussehen, erkennt man hier deutlich die in der fokalen Region erhöhten Verhältnisse der Konzentrationen $\mathrm{PCr} / \mathrm{P}_{\mathrm{i}}$ und $\mathrm{ATP} / \mathrm{P}_{\mathrm{i}}$, also die erhöhten Konzentrationen der energiereichen Verbindungen.

\section{Lit:}

1) Lauterbur P.C: Nature 242: 190, 1973

2) Damadian R: US Patent No. 3789832,1972

3) Kumar A., Welti D., Ernst R.R: J. Magn. Reson 18: 69, 1975

4) Bōsiger P: Kernspin-Tomographie für die med. Diagnostik,

B.G.Teubner, Stuttgart, 1985

5) Schoepflin R, Boesiger P: Biomed Techn (Erg.-Bd) 33, 1988

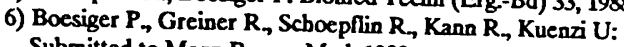

Submitted to Magn Reson Med, 1988

7) van Dijk P: J Comput Assist Tomogr 8(3):429, 1984

8) Meier D., Maier S, Boesiger P: Magn Reson Med 7, 1988

9) Aue W, Müller S., Cross T A., Seelig J: J Maga Res 56: 3560, 1984

10) den Hollander JA: Diagn Im Clin Med 55: 9, 1986

11) Ordidge RJ, Connelly A., Lohmann J.A.B: J Magn Res 66: 283, 1986

12) McKinnon G, Boesiger P: Magn Reson Med 6(3): 334, 1988

13) McKinnon G, Boesiger P: Biomed Techn (Erg.-Bd.) 33, 1988

14) Buchli R., Meier D, Boesiger P, Isler P, Wieser H.G: SMRM 1988

29 June, 1988 